Supporting information

\title{
for \\ Producing \\ Nanoporosities \\ in Block \\ Copolymers within 30 Seconds by \\ Microwave-Boosted Selective Swelling
}

Xiansong Shi, Xingyuan Wang, Yanjie Wang, and Yong Wang*

State Key Laboratory of Materials-Oriented Chemical Engineering and College of

Chemical Engineering, Nanjing Tech University, Nanjing 211816, P. R. China

E-mail: yongwang@njtech.edu.cn 


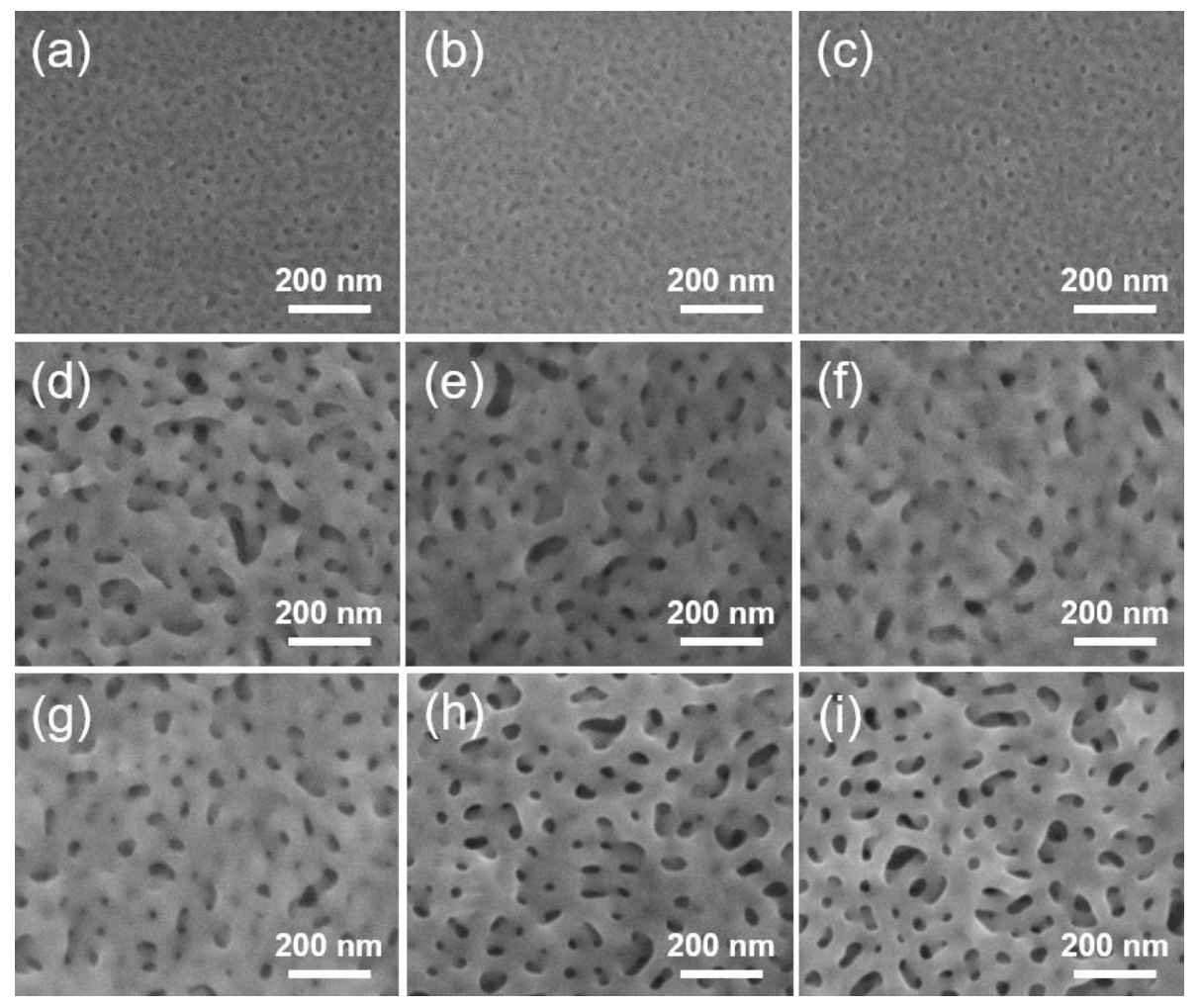

Figure S1. Surface morphologies of S2VP films after MBSS process. (a-c) Microwave swelling at $160 \mathrm{~W}$ for 20, 25, and 30 s, respectively; (d-f) microwave swelling at $400 \mathrm{~W}$ for 20,25 , and $30 \mathrm{~s}$, respectively; (g-i) microwave swelling at 800 W for 20, 25, and $30 \mathrm{~s}$, respectively.

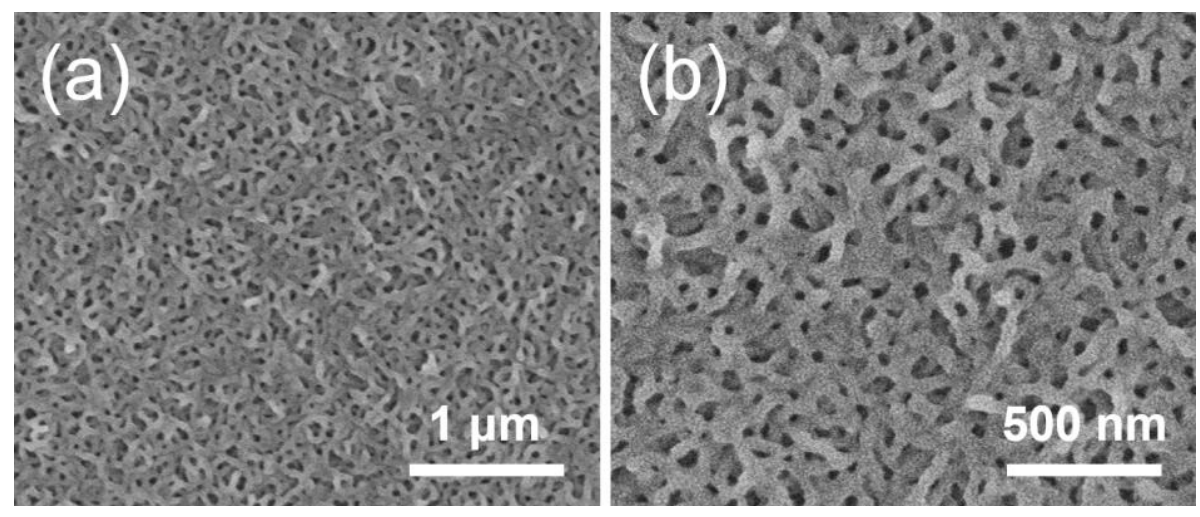

Figure S2. Surface morphologies of S2VP films after microwave swelling in acetic acid at $800 \mathrm{~W}$ for $20 \mathrm{~s}$. 


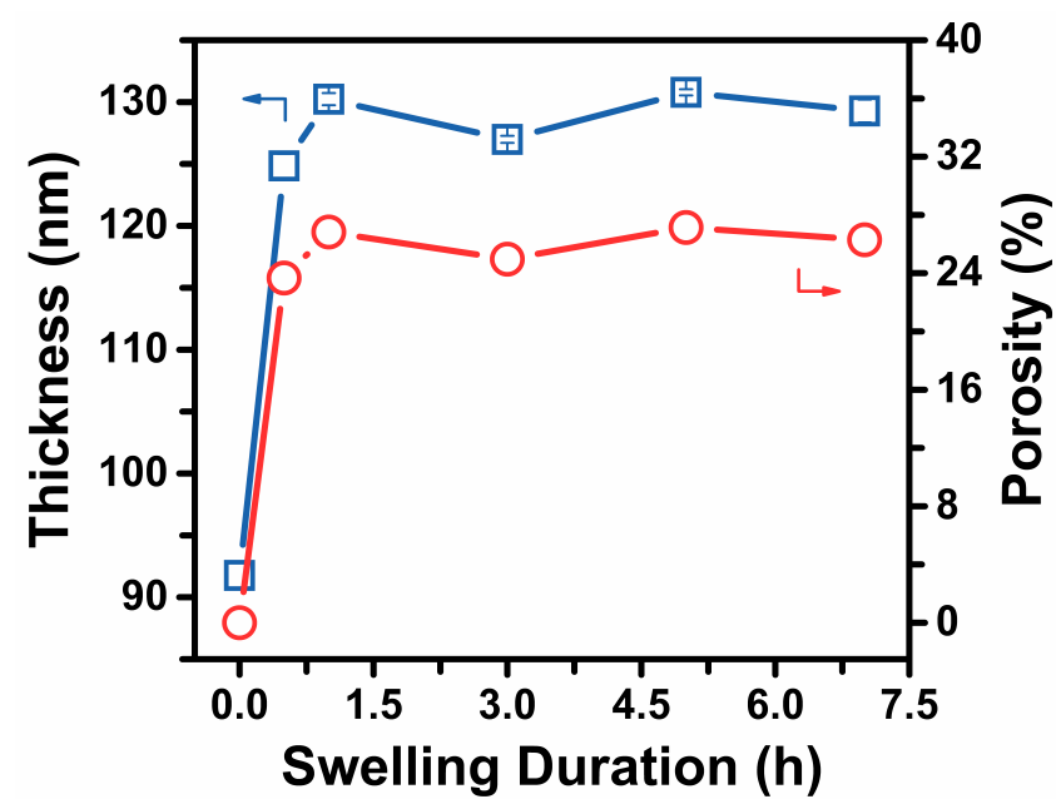

Figure S3. The change of the thickness and porosity after thermal swelling at $50^{\circ} \mathrm{C}$ for different hours.

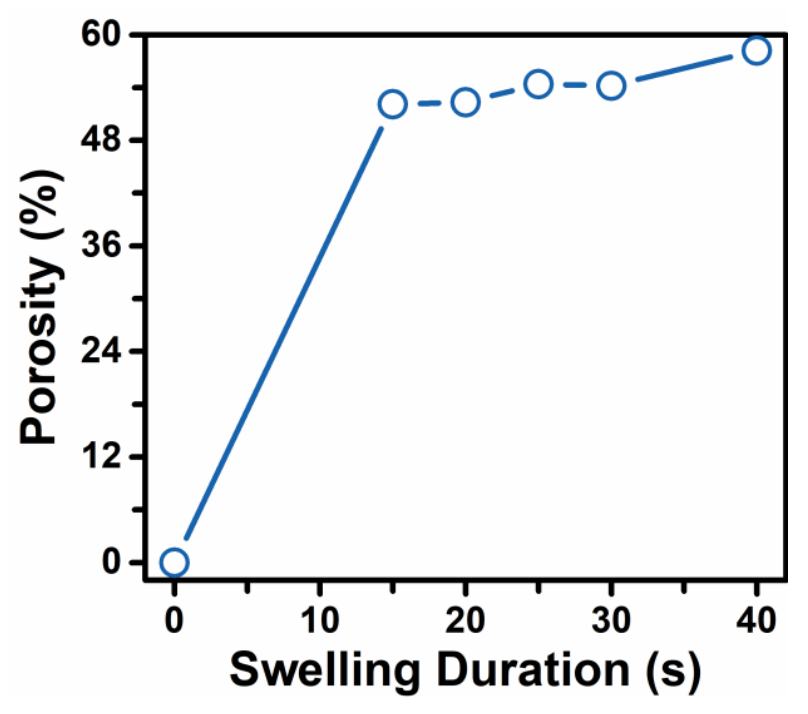

Figure S4. The change of porosity after microwave swelling at $400 \mathrm{~W}$ for different seconds. 
(a) + CH-CH ${ }_{\mathrm{m}} b+\mathrm{CH}_{2}-\mathrm{CH}_{2}-\mathrm{O}+\mathrm{T}_{\mathrm{n}}$

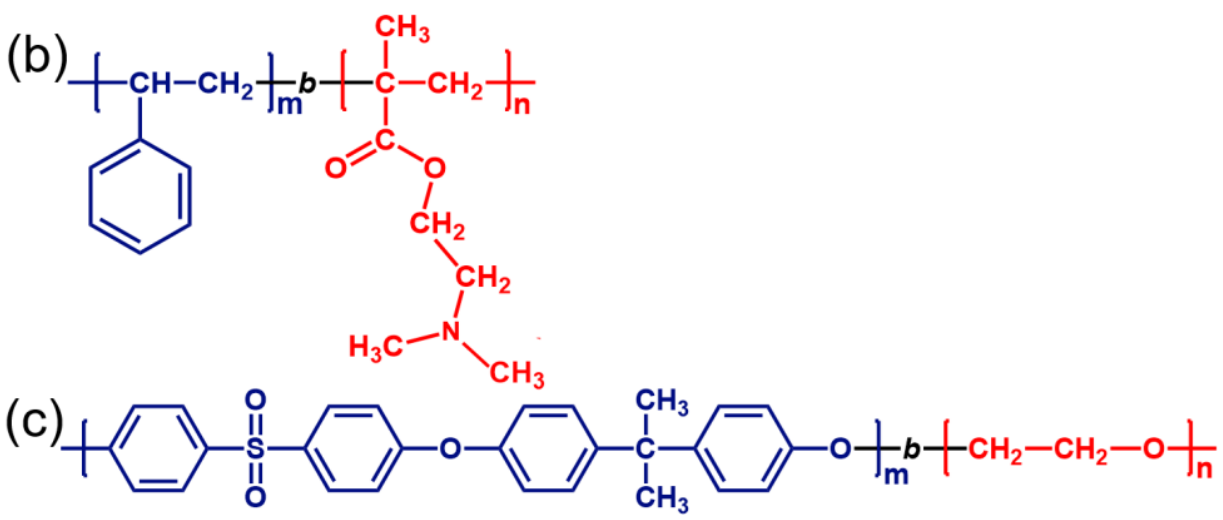

Figure S5. Molecular structures of SEO (a), SDAM (b), and SFEG (c).
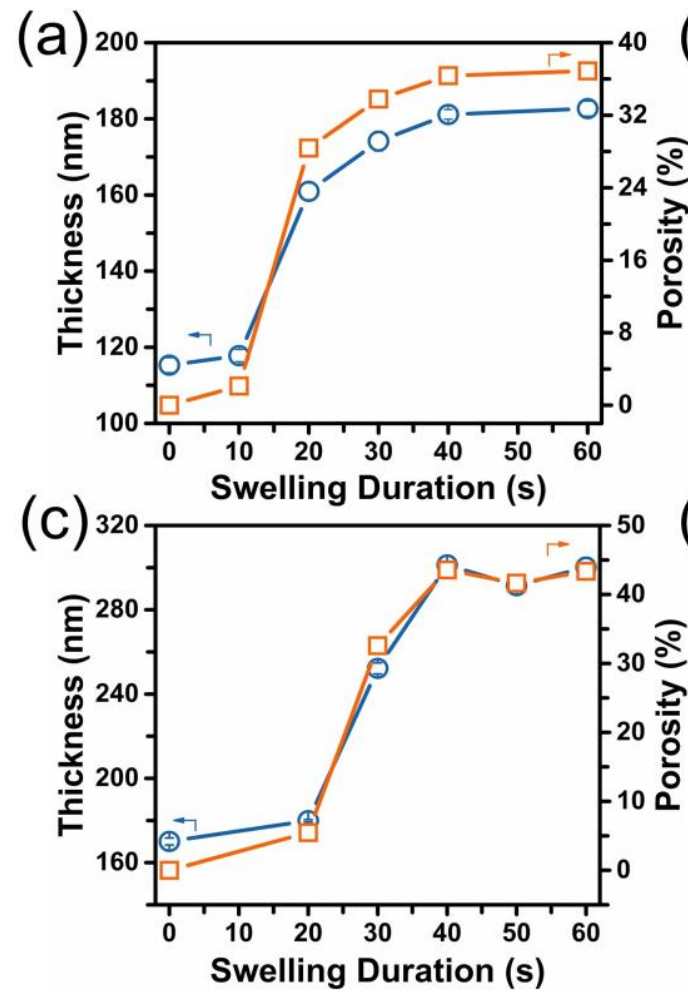

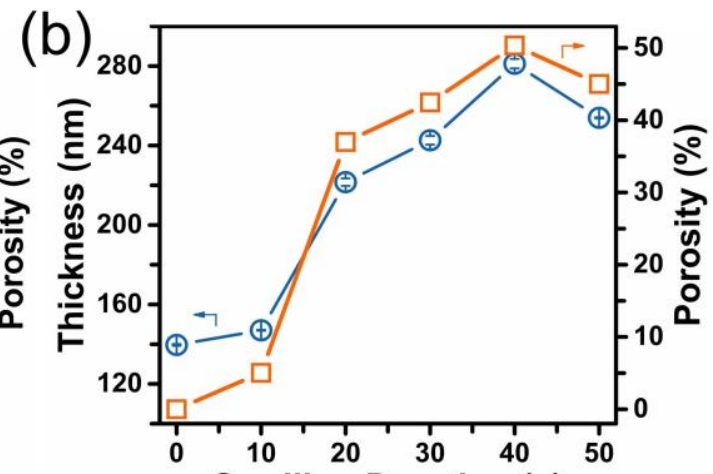

(d)

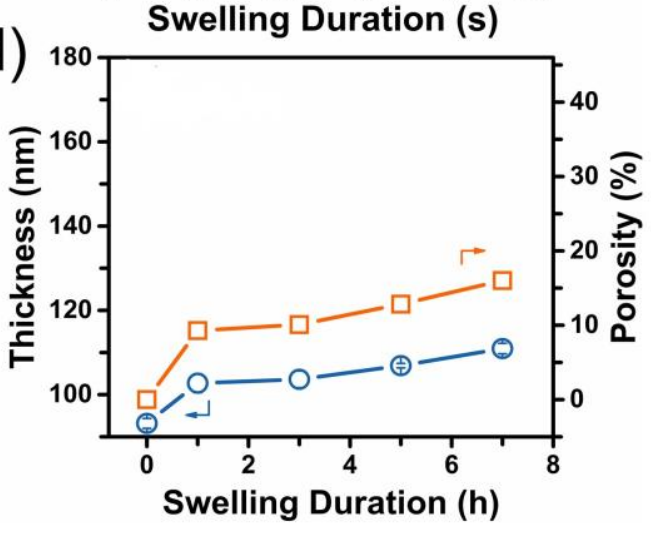

Figure S6. Selective swelling of other BCPs. (a-c) Thickness and porosity changes of

SEO (a), SDAM (b), and SFEG (c) films after microwave swelling at $800 \mathrm{~W}$ for different seconds; (d) the thickness and porosity change of SFEG films after thermal swelling at $50^{\circ} \mathrm{C}$ for different hours. 
Table S1. The required operation condition for the cavitation of BCPs by different methods.

\begin{tabular}{|c|c|c|c|c|}
\hline BCPs & Methods & Conditions & Total time & Reference \\
\hline PS- $b$-PLA & etching & $0.5 \mathrm{M} \mathrm{NaOH}$ & $90 \mathrm{~min}$ & $\mathrm{~S} 1$ \\
\hline PS- $b$-PI- $b$-PS- $b$-PLA & etching & $\begin{array}{c}0.5 \mathrm{M} \mathrm{NaOH} \\
\mathrm{UV}\end{array}$ & 1 month & $\mathrm{S} 2$ \\
\hline PS- $b$-PMMA & etching & $\begin{array}{l}\text { irradiation-acetic } \\
\text { acid }\end{array}$ & $4 \mathrm{~h}$ & S3 \\
\hline PS-r-PMMA/PMMA & dissolving & acetic acid & $1 \mathrm{~h}$ & S4 \\
\hline PS- $b$-P2VP & selective swelling & $60^{\circ} \mathrm{C}$, ethanol & $1-15 \mathrm{~h}$ & S5 \\
\hline PSF- $b$-PEG & selective swelling & $\begin{array}{c}70^{\circ} \mathrm{C} \\
\text { ethanol/acetone }\end{array}$ & $1 \mathrm{~h}$ & S6 \\
\hline PS- $b$-P2VP & MBSS & $400 \mathrm{~W}$, ethanol & $15-40 \mathrm{~s}$ & This work \\
\hline PS- $b$-PEO & MBSS & $800 \mathrm{~W}$, ethanol & $20-40 \mathrm{~s}$ & This work \\
\hline PS- $b$-PDMAEMA & MBSS & $800 \mathrm{~W}$, ethanol & $20-40 \mathrm{~s}$ & This work \\
\hline PSF- $b$-PEG & MBSS & $800 \mathrm{~W}$, ethanol & $20-40 \mathrm{~s}$ & This work \\
\hline
\end{tabular}
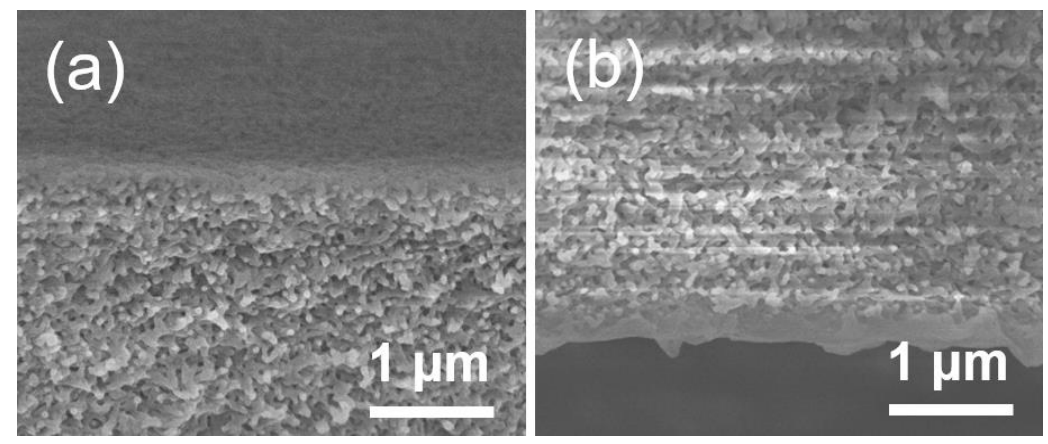

Figure S7. Cross-sectional morphologies of SFEG thin flat sheets after MBSS process. (a) Top area; (b) bottom area. 

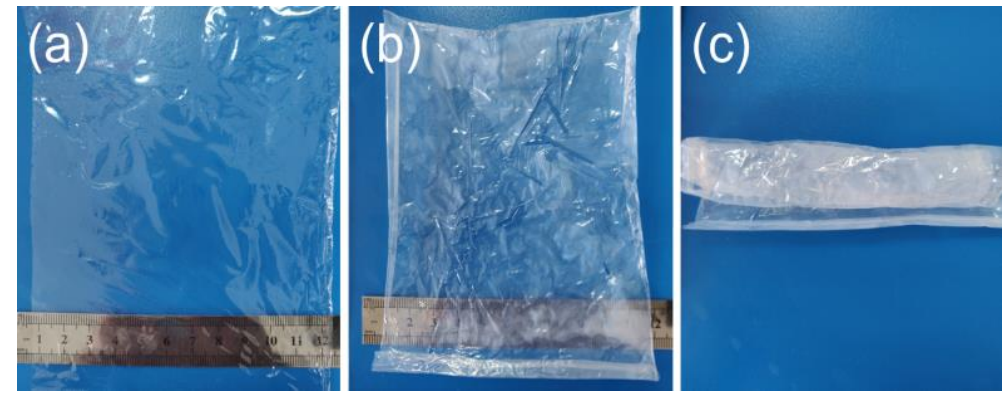

Figure S8. Digital images of large-size SFEG membranes before (a) and after (b, c)

MBSS process. (c) shows the fexibility of the produced porous membranes.
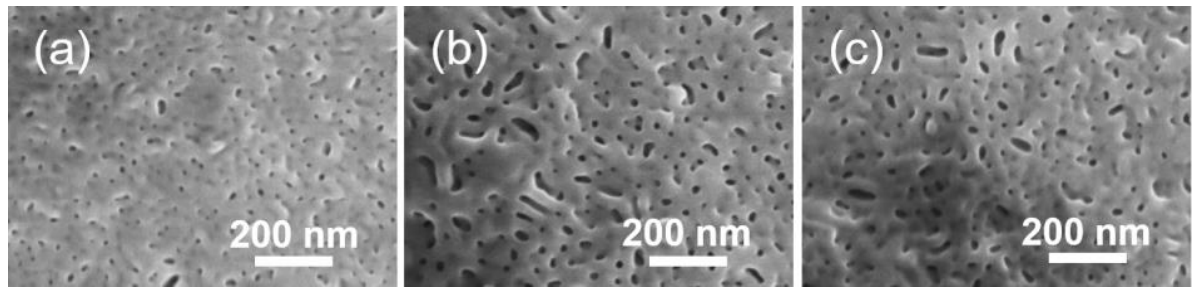

Figure S9. Surface morhologies of S2VP composite membranes after microwave swelling at $400 \mathrm{~W}$ for various seconds. (a) $15 \mathrm{~s}$; (b) $20 \mathrm{~s}$; (c) $25 \mathrm{~s}$.
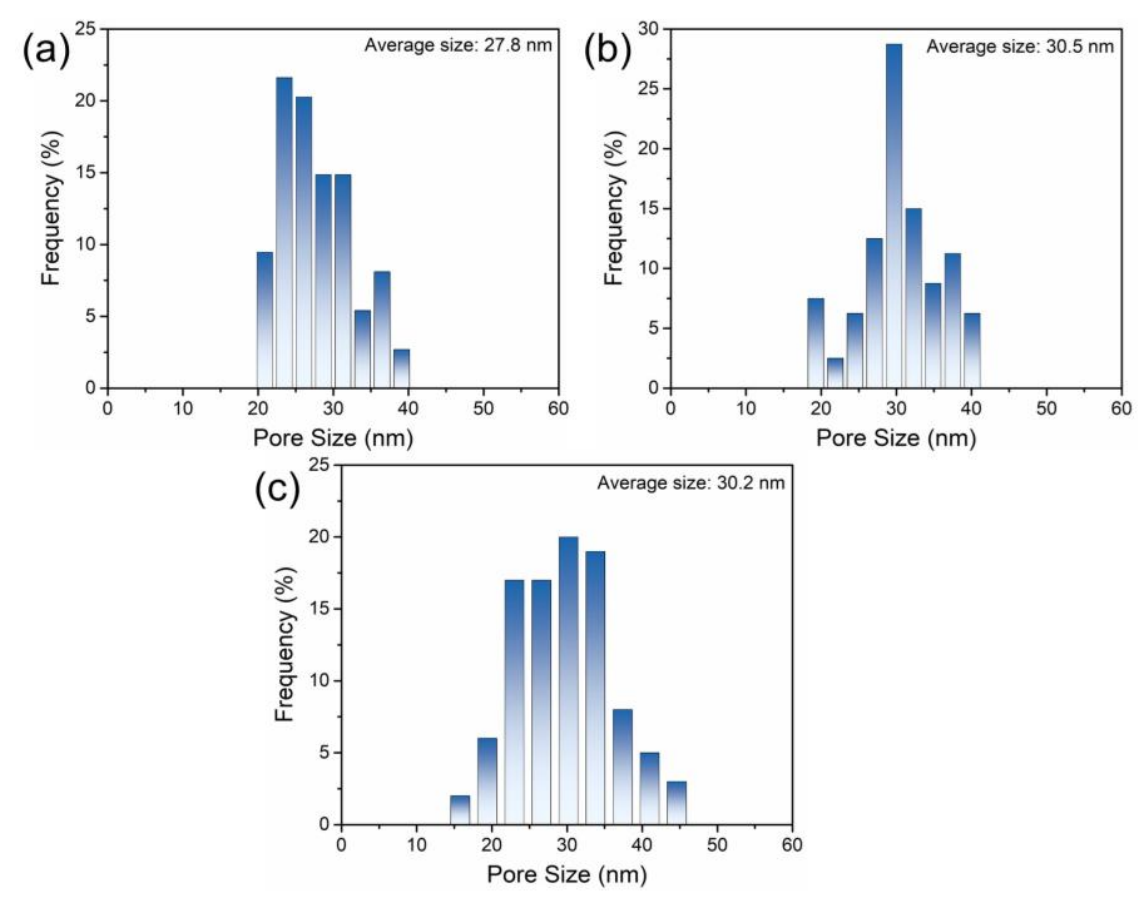

Figure S10. Size distributions of pores in different locations of the S2VP selective layer after microwave swelling at $400 \mathrm{~W}$ for $40 \mathrm{~s}$. (a) Top; (b) middle; (c) bottom. 


\section{REFERENCES}

(S1) Phillip, W. A.; O'Neill, B.; Rodwogin, M.; Hillmyer, M. A.; Cussler, E. L., Self-assembled block copolymer thin films as water filtration membranes. ACS Appl. Mater. Interfaces 2010, 2, 847-853.

(S2) Jackson, E. A.; Lee, Y.; Hillmyer, M. A., ABAC tetrablock terpolymers for tough nanoporous filtration membranes. Macromolecules 2013, 46, 1484-1491.

(S3) Yang, S. Y.; Park, J.; Yoon, J.; Ree, M.; Jang, S. K.; Kim, J. K., Virus filtration membranes prepared from nanoporous block copolymers with good dimensional stability under high pressures and excellent solvent resistance. Adv. Funct. Mater. 2008, 18, 1371-1377.

(S4) Yang, S. Y.; Ryu, I.; Kim, H. Y.; Kim, J. K.; Jang, S. K.; Russell, T. P., Nanoporous membranes with ultrahigh selectivity and flux for the filtration of viruses. Adv. Mater. 2006, 18, 709-712.

(S5) Wang, Y.; He, C. C.; Xing, W. H.; Li, F. B.; Tong, L.; Chen, Z. Q.; Liao, X. Z.; Steinhart, M., Nanoporous metal membranes with bicontinuous morphology from recyclable block-copolymer templates. Adv. Mater. 2010, 22, 2068-2072.

(S6) Wang, Z. G.; Liu, R.; Yang, H.; Wang, Y., Nanoporous polysulfones with in situ PEGylated surfaces by a simple swelling strategy using paired solvents. Chem. Commun. 2017, 53, 9105-9108. 